\title{
A DIMENSÃO PRÁTICA DO CUIDADO DE ENFERMAGEM: REPRESENTAÇÕES SOCIAIS DE ACADÊMICOS DE ENFERMAGEM ${ }^{a}$
}

\author{
The practical dimension in nursing care: Social Representations of Nursing Students. \\ La dimensión práctica de la atención de Enfermería: representaciones sociales de \\ académicos de enfermería
}

Natália Elisa Duarte

Márcia de Assunção Ferreira²

Márcia Tereza Luz Lisboa ${ }^{3}$

\section{RESUMO}

0 objetivo foi identificar os aspectos da dimensão prática do cuidado de enfermagem na perspectiva das representações sociais elaboradas por acadêmicos iniciantes e concluintes da graduação. 0 referencial teórico-metodológico foi 0 da Teoria das Representações Sociais. Tratou-se de uma pesquisa de abordagem qualitativa exploratória, com coleta de dados por entrevista individual com 28 acadêmicos, que sofreu análise temática de conteúdo. Os resultados demonstraram as peculiaridades dos elementos constituintes das representações sociais elaboradas pelos dois grupos. As representações sociais dos dois grupos se distinguem, já que os iniciantes descrevem uma prática voltada aos aspectos afetivo-expressivos, enquanto os concluintes trazem marcadamente a conjugação desses aspectos com o conhecimento científico. Conclusão: 0 curso de graduação, gradativamente, influencia nas representações sociais do cuidado e, consequentemente, na sua dimensão prática.

Palavras-chave: Enfermagem. Cuidados de enfermagem. Psicologia social.

\begin{abstract}
This study aims to identify aspects of the practical dimension in nursing care under the perspective from the social representations elaborated by nursing students beginning the graduation, and others about to conclude it. The theoretical-methodological reference was the Theory of Social Representations. This was an exploratory qualitative study, collecting data through individual interviews applied to 28 students which had a thematic content analysis. The results showed the peculiarities of the constituent elements of social representations made by both groups. Social representations of both groups are distinguished, as the beginners describe a practice focused on affective and expressive aspects markedly while the students about to be graduated bring, effectively, a combination of these aspects with scientific knowledge. Conclusion: The graduation program gradually influences the social representations in care, and consequently in its practical dimension.
\end{abstract}

Keywords: Nursing. Nursing Care. Psychology Social.
Resumen

Objetivo: identificar los aspectos de la dimensión práctica de la atención de enfermería desde la perspectiva de las representaciones sociales elaboradas por académicos juniores y concluyentes de la graduación. El referencial teórico y metodológico fue el de la Teoría de las Representaciones Sociales. Es una investigación de abordaje cualitativo exploratorio, con recolección de datos a través de entrevista individual con 28 académicos, y ha sido realizado el análisis temático de contenido. Los resultados demostraron las peculiaridades de los elementos constituyentes de las representaciones sociales elaboradas por dos grupos. Las representaciones sociales de los dos grupos se distinguen, ya que los juniores describen una práctica involucradas a los aspectos afectivos y expresivos, mientras los concluyentes trajeron marcadamente la conjugación de eses aspectos con el conocimiento científico. Conclusión: El curso de graduación, progresivamente, influencia en las representaciones sociales de la atención, y, consecuentemente, en su dimensión práctica.

Palabras claves: Enfermería. Atención de enfermería. Psicología Social.

\footnotetext{
${ }^{1}$ Mestre em Enfermagem. Aluna do curso de doutorado do Programa de Pós-Graduação da Escola de Enfermagem Anna Nery, Universidade Federal do Rio de Janeiro (EEAN-UFRJ). Membro do Núcleo de Pesquisa de Fundamentos do Cuidado de Enfermagem. Bolsista CNPq (2008-2010). Rio de Janeiro - RJ. Brasil. E-mail: naty_ufrj2003@yahoo.com.br; ²Doutora em Enfermagem. Professora Titular do Departamento de Enfermagem Fundamental da Escola de Enfermagem Anna Nery, Universidade Federal do Rio de Janeiro. Pesquisadora do CNPq. Rio de Janeiro - RJ. Brasil. E-mail: marciadeaf@ibest.com.br; ${ }^{3}$ Doutora em Enfermagem. Professora Associada do Departamento de Enfermagem Fundamental da Escola de Enfermagem Anna Nery, Universidade Federal do Rio de Janeiro. Membro do Núcleo de Pesquisa de Fundamentos do Cuidado de Enfermagem. Rio de Janeiro - RJ. Brasil. E-mail:marcialis@terra.com.br
} 


\section{INTRODUÇÃO}

0 cuidado de enfermagem resulta da ação de enfermagem. No seu processo de trabalho, esta ação se materializa no cuidado do ser humano (cliente da enfermagem). Portanto, seu objetivo é a promoção da saúde, a prevenção de doenças, a recuperação e a reabilitação da saúde, sendo considerado como a função precípua do enfermeiro!.

Neste estudo, o fenômeno social em foco é o cuidado de enfermagem, que tem espessura social, isso é, tem relevância para um determinado grupo, já que as representações sociais sobre o cuidado de enfermagem formam um conjunto de ideias que guiam as ações daqueles que o exercem e uma reação daqueles que o recebem.

0 cuidado humano é um ato que exprime a necessidade do encontro de duas pessoas em uma situação ou em um determinado ambiente. Ao considerar o significado do cuidado como desvelo, solicitude, diligência, zelo, atenção, percebe-se que ele integra o processo de sobrevivência humana, tratando-se de um valor intrínseco da vida².

$\mathrm{Na}$ enfermagem, o cuidado é realizado no encontro entre quem o realiza e quem o recebe. A sua prática é singular e se dá no próprio ato. Neste sentido, é pertinente afirmar que os acadêmicos iniciantes, que estão tendo o primeiro contato com o cuidado profissional, elaborarão suas representações a fim de tornar familiar aquilo que the é estranho. E o grupo social dos acadêmicos concluintes, que já teve contato com os conteúdos formais da graduação, formula suas representações com base nas experiências práticas, teóricas, sociais, familiares, entre outras.

Àluz deste entendimento, o cuidado de enfermagem, na medida em que provoca repercussões tanto em quem 0 realiza quanto em quem o recebe, e na própria prática do enfermeiro, produz informações que circulam no grupo e que explicam sua forma de pensar e agir.

0 problema delineado foi: Quais são as representações sociais (RS) de acadêmicos de enfermagem sobre o cuidado de enfermagem e como os acadêmicos se comportam diante do cuidado? Os objetivos da pesquisa foram: identificar as RS do cuidado de enfermagem e descrever os aspectos da dimensão prática do cuidado de enfermagem, diante das representações sociais elaboradas por acadêmicos iniciantes e concluintes do curso de graduação em enfermagem.

\section{BASES CONCEITUAIS}

0 cuidado de enfermagem é a essência da profissão, que apresenta uma vertente objetiva referente ao desenvolvimento de técnicas e procedimentos, e uma vertente subjetiva balizada pela sensibilidade, criatividade e intuição?

0 trabalho de enfermagem visa à integralidade do sujeito, consequentemente o cuidado deve conjugar a arte e a técnica, por isso é possível determinar que a sua execução requer conhecimento do outro, sobre o outro e sobre nossas competências enquanto enfermeiros ${ }^{3}$.

Assim sendo, o cuidado de enfermagem não deve se desvincular do seu lado humano, mesmo com os avanços tecnológicos, que podem levar à carência das expressões humanas. Deve-se considerar que 0 ato de cuidar implica 0 estabelecimento de interação entre sujeitos (quem cuida e quem é cuidado) que participam da realização de ações e que isso é a verdadeira essência da enfermagem ${ }^{4}$.

A academia deve possibilitar a interação do acadêmico com a população dentro do seu contexto social e a cooperação estudante-docente para assim permitir o desenvolvimento da autonomia e facilitar o processo de ensino-aprendizagem ${ }^{5}$.

Outra observação relevante refere-se à necessidade de compartilhamento de saberes em uma construção coletiva entre a prática em saúde e o processo ensino-aprendizagem ${ }^{6}$.

\section{REFERENCIAL TEÓRICO- METODOLÓGICO}

Pesquisa descritiva com abordagem qualitativa exploratória, com referencial teórico-metodológico da Teoria das Representações Sociais (TRS) ${ }^{7}$. As representações sociais (RS) têm função constitutiva da realidade, da única realidade que conhecíamos por experiência e na qual a maioria das pessoas se movimenta?

Por isso, as RS orientam e organizam as condutas e comunicações sociais e intervêm em processos variados, tais como difusão e assimilação dos conhecimentos, o desenvolvimento individual e coletivo, a definição das identidades pessoais e sociais, a expressão dos grupos e as transformações sociais ${ }^{8}$.

$\mathrm{Na}$ TRS, o objeto de pesquisa deve ter requisitos conceituais adequados à teoria para instruí-lo. Além disso, deve ser algo que possua relevância social e acadêmica.

0 cuidado de enfermagem e a sua dimensão prática compõem o corpo de conhecimento da enfermagem, e importam não só aos enfermeiros que as executam, mas também a população que é atendida por eles. Em consequência, a representação construída acerca dele, que é objeto de trabalho da enfermagem, influencia a prática profissional e supõe, entre outras coisas, o reconhecimento do que seja concebido como cuidado de enfermagem.

A fim de apreender a percepção subjetiva dos atores sociais foi utilizada uma abordagem compreensiva qualitativa, que possibilita acessar a elaboração das RS dos acadêmicos, de acordo com sua percepção e vivências.

0 cenário foi um Curso de Graduação em Enfermagem de uma Universidade Federal. Participaram da pesquisa 28 acadêmicos, sendo 14 iniciantes e 14 concluintes. Os critérios de inclusão foram: ser acadêmico de enfermagem; estar cursando os dois primeiros e os dois últimos meses do curso de graduação; aceitar participar da pesquisa. 
Os dados foram coletados por meio de entrevista individual, com roteiro de questões semiestruturadas, registrada digitalmente em formato $\mathrm{mp} 3$ e transcrita integralmente.

Aplicou-se aos dados a técnica de análise de conteúdo temático. Primeiramente foi realizada análise separadamente do corpus de dados obtidos de cada um dos grupos de acadêmicos, e depois foi realizado o levantamento das aproximações e distanciamentos a fim de captar os aspectos da dimensão prática presentes na construção das representações sociais e as peculiares de cada um dos grupos de acadêmicos de enfermagem.

A Resolução 196/96 foi atendida, e o projeto foi aprovado pelo Comitê de Ética em Pesquisa EEAN/HESFA, protocolo n033/2009, e todos os acadêmicos assinaram 0 Termo de Consentimento Livre e Esclarecido. A coleta de dados dos acadêmicos iniciantes ocorreu nos dois primeiros meses do curso em agosto e setembro de 2009, e dos concluintes ocorreu nos dois últimos meses do curso em novembro e dezembro de 2009.

\section{RESULTADOS E DISCUSSÃO}

\section{Iniciantes: o cuidado afetivo inter-relacional}

Este grupo, ao construir sua representação do cuidado de enfermagem, tendo como base a representação do cuidado humano e a imagem do profissional enfermeiro, aponta 0 cuidado de enfermagem traduzido como prática interativa, isso é, que só ocorre na presença de uma relação afetiva com o cliente.

Tem que ter a relação com o paciente porque fazer o cuidado de enfermagem sem ter aquela relação com o paciente, fazer qualquer coisa por fazer, ou não fazer, dormir durante um plantão, e deixar o paciente sozinho por causa do sono, não é cuidado de enfermagem. (E11)

Logo, pode-se intuir que a RS sobre a prática do cuidado de enfermagem foi elaborada a partir das vivências sobre o cuidado ao longo da vida, das crenças particulares, das influências externas e informações da mídia. Em consequência, esta RS orienta a prática de um cuidado de enfermagem a ser executado, considerando os sentimentos dos sujeitos.

Dentro do ambiente acadêmico estes sujeitos terão a oportunidade do contato com o cuidado de enfermagem que é peculiar dessa profissão, e, por este ser o objeto de trabalho profissional, é necessário considerar os aspectos que permeiam essa prática. Assim, o acadêmico iniciante irá lidar com os saberes científicos que lhe são apresentados e mobilizar os saberes sociais prévios sobre o cuidado humano e os afetos da clientela com a qual deverá estabelecer uma relação de cuidado (conteúdo teórico e prático do cuidado).
Encontraram-se os seguintes elementos no discurso dos acadêmicos iniciantes sobre a prática do cuidado de enfermagem: cuidado humano, relação enfermeiro-cliente e tratar bem o paciente.

0 envolvimento do enfermeiro com o cliente no seu aspecto humano é descrito pelos acadêmicos com a expressão "estar presente", como pode ser observado no trecho a seguir:

Éestar presente, e passar segurança para o paciente e não perder suas obrigações como enfermeiro, 0 que você aprendeu, o que você tem que exercer, $e$ saber na hora de fazer. (E5)

Além disso, há expressões dos iniciantes relacionadas ao cuidado como a forma de tratar (bem) e se relacionar com o outro, o bom trato dado pelo profissional:

[Cuidado de enfermagem é] chegar e falar se está tudo bem, não que chegou cedo e tal, ser humano. Ser humano pra mim é a gente tratar bem as pessoas, tratar bem é você fazer o seu melhor para aquela pessoa passar bem. (E1)

Ressaltaram-se as seguintes co-ocorrências: atenção e carinho; atenção e dedicação; e carinho e dedicação. Consequentemente, o cuidado de enfermagem torna-se uma prática viabilizada pelas relações humanas na qual o profissional deve estabelecer com o cliente uma relação de confiança, a fim de exercer suas obrigações. Tal resultado é recorrente nos estudos de enfermagem que investem em aprofundar as bases nas quais se assentam o cuidado. Sendo o cuidado fruto da ação da enfermeira, envolve relação e interação, nas quais não se pode prescindir das qualidades humanas ${ }^{2,4,9}$.

0 cuidado de enfermagem distingue-se do cuidado humano por ser dotado de conhecimentos científicos e tem como objetivo a melhoria do paciente, incluindo a própria cura.

[Cuidado de enfermagem] Tem essa atenção, essa preocupação, mas tem o tratamento, tem os procedimentos que não é qualquer um que pode fazer, tem que ter um conhecimento para isso, uma prática. (E10)

Na unidade de registro exemplificada, os elementos atenção e preocupação aliam-se aos procedimentos e tratamento. No entanto, há indício de que, na RS comunicada, estes dois últimos elementos são exclusivos do constructo cuidado profissional, pois exigem conhecimento (técnico e científico); já os dois primeiros, constituem tanto o cuidado humano (leigo) quanto o profissional. Esta análise foi possível por meio da interpretação sobre como se deu a construção do pensamento de E10, relacionando os dois elementos em primeira instância, seguida da conjunção 'mas', que expressa 
uma ressalva inclusiva em relação aos outros dois elementos seguintes, relativos ao objeto que lhe foi dado a pensar: 0 'cuidado de enfermagem'.

E ainda, os acadêmicos iniciantes, ao reconhecerem uma prática na enfermagem desprovida do cuidado de enfermagem, utilizam-se da expressão "qualquer coisa" referindo-se aos procedimentos e às relações. Portanto, trazem o conceito de que se não há 'cuidado de enfermagem' (com elementos que o caracterizem - próprio dela), a prática não é 'de' enfermagem, mas sim, 'qualquer prática - cuidado leigo', comunicada na expressão consensual "qualquer coisa”. Logo, não é prática 'de', mas sim prática 'na'. Observa-se esse movimento da construção do pensamento a seguir:

É chegar dando atenção, dar carinho, não chegare fazer qualquer coisa, de qualquer jeito, ter o cuidado também, na hora de fazer, fazer direito as coisas de enfermagem mesmo. (E4)

As atitudes de cuidado identificam a essência da expressão humana, uma vez que apenas este ser está capacitado a sentir, imprimir e expressar emoções, compreendendo-as como tomada de posição resultante da inter-relação entre 0 conhecimento e o afeto $^{9}$.

Ainda, são observáveis alguns aspectos da dimensão desta prática, como: assistir ao outro, envolver-se, estar presente, confortar, preocupar-se, ter consideração, ter compaixão, expressar sentimentos, fazer para/com, respeitar, ter habilidade técnica, demonstrar conhecimento e segurança ${ }^{10}$.

Assim sendo, a prática do cuidado de enfermagem para esse grupo é algo espećfico da profissão, deve ser realizada segundo as funções do enfermeiro. Esta prática reune técnicas e procedimentos baseados no conhecimento profissional; porém, o mais marcante desta ação é o conjunto de suas características humanísticas.

Esse grupo de acadêmicos, ao relacionar os aspectos técnicos e humanos na prática do cuidado de enfermagem, realça a importância da relação estabelecida entre o profissional e o cliente. Refletindo-se sobre essa relação estabelecida, percebese que ocorre nela um envolvimento humano que inclui a equipe de saúde e a instituição, contemplando elementos administrativos e organizacionais, os quais contribuem para incremento da complexidade do contexto ${ }^{11}$.

Consequentemente, o objetivo do cuidado de enfermagem é fazer com que o paciente se sinta melhor, já que o bem-estar do cliente é uma resposta cujos elementos compõem a ideia sobre o cuidado de enfermagem. Consequentemente, 0 cuidado de enfermagem não é por si, mas se define pela resposta do cliente.

Cuidado de enfermagem é aquilo, você fazer com que aquela pessoa, que você no caso está cuidando, se sinta melhor. (E4)
Os resultados evidenciados mostram-se relevantes na medida em que o cuidado é compreendido como um elemento indispensável nas relações humanas para o bem-estar, manutenção da saúde e da própria vida ${ }^{12}$.

A enfermagem possui um saber-fazer espećifico que se traduz em seu conhecimento e em sua ação, os acadêmicos descreveram essa prática determinando uma maneira pela qual o cuidado deve ser executado. Essa forma de realizar o cuidado de enfermagem se dá pelo conhecimento específico da profissão, quanto aos aspectos referentes ao cliente e aos procedimentos.

Observa-se, portanto, que os acadêmicos identificam práticas bem feitas e mal feitas e estas formas de agir os levam a classificar os cuidados pela ação: bem ou mal feito. Consequentemente, o grupo de acadêmicos identifica que o cuidado é bem feito quando, na sua prática, o enfermeiro realiza suas obrigações através do conhecimento profissional, enquanto o cuidado mal feito é aquele em que o enfermeiro não possui o conhecimento necessário para realizar suas obrigaç̃̃es. Neste ínterim, as representações sociais do cuidado nos levam a identificar que os acadêmicos mobilizam os conhecimentos do senso comum, em termos da polaridade bem e mal, para classificar as ações de cuidado. Estes resultados apontam, portanto, a necessidade de se investir mais efetivamente no ensino do conceito de cuidado profissional que, por si, é ético. Logo, à luz do conceito, não existiria cuidado mal feito, pois o seu contrário seria o descuido (ação mal feita), o não-cuidado.

Sobre a prioridade do cuidado de enfermagem, esta está em realizar tudo aquilo que gerará melhora para o paciente, conjugando o fazer do profissional com uma forma específica de fazê-lo. Essa forma de realizar o cuidado de enfermagem é permeada por aspectos afetivos, de entrega pela vida do outro. 0 uso dos sentidos humanos, bem como o modo como são aplicados, é destacado: forma de falar, maneira de se relacionar com o cliente, com atenção e respeito. Estes elementos dão forma à prática do cuidado de enfermagem, atribuindo-lhe grau de importância. Porém, os acadêmicos não identificam um cuidado que seja menos importante, e isto leva à inferência de que ainda não desenvolveram a condição de determinar claramente a existência de uma hierarquia estruturada.

\section{Concluintes: o cuidado profissional}

Este grupo construiu sua representação sobre 0 cuidado de enfermagem, na ideia de cuidado humano como atitude, e na concepção de que o enfermeiro é um profissional que tem um campo de conhecimento científico específico baseado em pesquisas, o qual é empregado no momento do cuidado. Pode-se entender, então, que, para exercer o cuidado de enfermagem, é necessário ter conhecimentos científicos e saber utilizá-los:

Porque [para fazer o cuidado de enfermagem] jáé preciso, de respaldo teórico-prático, ter 
conhecimento daquilo que eu faço, eu vou interferir e posso prejudicar uma pessoa se eu não souber o que estou fazendo. (E18)

A realização do cuidado de enfermagem se dá no desenvolvimento de ações fundamentadas no conhecimento científico, habilidade, intuição, pensamento crítico e criatividade, associadas aos comportamentos e atitudes que visam recuperar a totalidade e a dignidade humana ${ }^{13}$.

A dimensão prática do cuidado situa-se na sua própria essência, pois para os concluintes, o cuidado de enfermagem trata-se da realização de ações que visam responder a uma necessidade do outro, seja ela uma necessidade física, psicológica ou emocional, ou ainda um desejo. Por isso, realizar essa prática sempre dependerá do sujeito com e para quem está sendo executado o cuidado.

Os acadêmicos descrevem que o cuidado de enfermagem é realizado a partir de um planejamento, o que pode indicar que esses acadêmicos utilizam-se do conhecimento científico obtido durante o curso de graduação quanto ao Processo de Enfermagem, acrescentando este elemento ao campo de sua representação social sobre o cuidado, o que influenciará a sua prática. Já que o processo de enfermagem, ou a sistematização da assistência de enfermagem, pressupõe uma série de ações dinâmicas e inter-relacionadas para sua realização, ou seja, indica a adoção de um determinado método ou modo de fazer ${ }^{14}$.

Consequentemente, o cuidado só poderá ser exercido quando houver uma interação que possibilite conhecer quais são as expectativas do sujeito a respeito do cuidado de enfermagem. Porém, os concluintes ainda afirmam que a própria interação, na medida em que promove a participação do outro, também é um cuidado.

Foi identificado pelo grupo que a sua prática respeita a individualidade do cliente já que aponta como prioridade o atendimento das necessidades e desejos para e com o qual está sendo realizado o cuidado, segundo o que o mesmo determina.

Para que o paciente se sinta realmente cuidado, são necessárias informação e atenção a ele, e ao que estiver a ele relacionado durante a prática da equipe de saúde ${ }^{12}$, o que reafirma a interação como algo inerente ao cuidado, tanto para os enfermeiros quanto para os clientes, e reitera os resultados encontrados na pesquisa em tela.

Outros aspectos da prática do cuidado levantados pelos concluintes foram: estar presente; preocupar-se; ter consideração; e expressar sentimentos, sendo possível determinar as co-ocorrências em ordem decrescente de frequência: atenção e carinho; atenção e estar presente; estar presente e carinho; estar presente e preocupação; e preocupação e carinho. Esses dados vão ao encontro dos construtos de cuidar/ cuidado os quais consideram que as pessoas expressam, de acordo com seus padrões culturais, os comportamentos de cuidar que são desenvolvidos por elas ${ }^{10}$.
Portanto, para este grupo, a prática do cuidado se dá como uma forma de satisfazer as necessidades do outro e permite a articulação do saber do universo reificado sobre o cuidado de enfermagem com as suas experiências.

Na análise dos dados, detectou-se que os acadêmicos concluintes descrevem que o cuidado de enfermagem deve proporcionar condições para a melhoria do estado de saúde do outro, e em uma perspectiva ainda mais abrangente, buscar benefícios, através da oferta de ferramentas, para a família e a comunidade.

Uma característica marcante da prática do cuidado de enfermagem apontada por esses acadêmicos é a resolutividade, o cuidado como uma ação que é objetiva, resolutiva e que tem qualidade.

Esses discentes descrevem dois tipos de ações de cuidado: uma realizada junto ao cliente (cuidado direto) e outra realizada longe dele (cuidado indireto). Nesta classificação, os cuidados diretos são ações técnicasprocedimentais, enquanto os cuidados indiretos são todas as ações realizadas que fazem parte das responsabilidades do enfermeiro, mas que não implicam contato diretor com o cliente, tais como: as inerentes à gerência do cuidado e à administração do setor. Porém, foi descrito um pré-requisito para que a ação realizada seja considerada como um cuidado de enfermagem: proporcionar benefício ao cliente.

Logo, para os concluintes, quando o enfermeiro realiza uma ação que não visa a melhoria do estado de saúde do cliente, ele está realizando uma prática desprovida de elementos que caracterizam o cuidado como 'de enfermagem'.

Esses acadêmicos consideram como mais importante na realização do cuidado de enfermagem os elementos pertinentes à relação estabelecida entre 0 enfermeiro e 0 cliente, sendo identificadas as seguintes co-ocorrências: conhecer o outro, saber se relacionar com o outro, e saber ouvir o outro. Isso se deve ao fato de os acadêmicos determinarem que a prioridade do cuidado esteja em satisfazer as necessidades do sujeito.

Quando questionados sobre os cuidados mais e menos importantes, afirmaram não existir esse tipo de classificação; portanto, não há, a priori, esta classificação, mas ela ocorre na medida em que a prioridade é determinada pela necessidade ou situação na qual se encontra o cliente.

Pode-se identificar que este grupo de acadêmicos produziu sua representação social da prática do cuidado de enfermagem ao associarem informações de suas experiências como estudantes nas atividades práticas com a clientela, através de informações de experiências pessoais e sociofamiliares.

Os concluintes reiteram o cuidado de enfermagem como objeto da prática, tendo este um planejamento, um objetivo e uma forma específica de ser realizado. Por isso, é possível dizer que os acadêmicos reconhecem a compaixão como uma atitude de cuidado e de zelo com o outro, e, ainda, 
que seja necessário o conhecimento para execução do cuidado de enfermagem.

\section{Iniciantes versus Concluintes}

Os resultados apresentados permitem realizar aproximaç̃̃es e distanciamentos entre as representações construídas pelos dois grupos de acadêmicos.

0 processo de construção das representações sociais envolve dois mecanismos, ancoragem e objetivação, pelos quais o grupo social torna o "novo" familiar ${ }^{15}$. Identifica-se que os acadêmicos iniciantes utilizam os sentidos atribuídos ao cuidado, ou seja, ao cuidado humano e às suas experiências pessoais, para construir suas representações, o que imbrica na ideia préconcebida de preocupação com o outro, de zelo e de ações com aspectos afetivos.

Esses apontamentos permitem dizer que os elementos afetivos relacionados à origem da expressão "cuidado de enfermagem", isto é, relativos à própria palavra "cuidado", estavam presentes na construção das representações sobre o cuidado de enfermagem. E, dessa forma, agem como subsídios para a forma de pensar e de agir na prática do cuidado de enfermagem dos acadêmicos, tanto em fase inicial quanto em fase final do curso de graduação.

Portanto, o cuidado terá a função de ser o elo entre seres cuidados e seres cuidadores, que for talece e potencializa o processo de recuperação na condição de saúde-doença, interrelacionando os demais processos dinâmicos manifestos no ato de cuidar ${ }^{12}$.

Porém, para construir suas representações sobre o cuidado de enfermagem, os grupos o associam à questão profissional de formas diferentes, já que os iniciantes utilizam 0 aspecto profissional voltado às suas obrigações, enquanto os concluintes referem-se aos conhecimentos científicos profissionais e aos aspectos éticos do cuidado de enfermagem.

Outra aproximação é o fato de o cuidado de enfermagem ter seu foco no sujeito do cuidado, sendo sempre realizado para seu benefício. Entretanto, o grupo dos iniciantes diz que o cuidado de enfermagem é realizado para o sujeito, já o grupo dos concluintes diz que o cuidado de enfermagem é realizado para e com o sujeito. Neste último, identifica-se a forte influência de elementos afetivos e éticos, no sentido de que 'fazer com' implica considerar o outro na relação, ou seja, os concluintes incluem o cliente como elemento do cuidado profissional, pois a ação centra-se em ambos os implicados nela: profissional e cliente. Os iniciantes centram a ação no agente (profissional), pois a ação parte dele para o outro.

Essas similaridades e diferenças de cada grupo social de acadêmicos permitem a construção de representações sociais que refletem influências do cuidado humano e da academia. Os acadêmicos do primeiro período, por não dominarem as funções do enfermeiro e por não terem tido contato com a prática desse cuidado, utilizam-se da noção prévia do que seja um profissional de saúde como aquele que exerce suas funções de forma correta, ou como eles mesmos relataram: 'da melhor forma possível'. $\mathrm{E}$ a isso conjugaram o cuidado humano, que é aquele em que o ser que cuida se preocupa e zela pelo outro, demonstrando aspectos afetivos.

Os acadêmicos do último período, já tendo sofrido influências da academia de forma mais profunda, por terem passado quatro anos experienciando e vivenciando a prática do cuidado de enfermagem, construíram sua representação na articulação do cuidado humano com o que identificaram ser peculiar na formação acadêmica: o conhecimento científico e ético.

Ao analisar os discursos dos acadêmicos à luz da Teoria das Representações Sociais, considera-se que as RS são compreendidas nos grupos sociais, o que impede sua generalização; logo, os membros do grupo, ao falarem sobre um objeto, comunicam as formas de pensá-lo e as de agir de outros membros do mesmo grupo, já que possuem as mesmas características socioculturais e condições de produção.

Por isso, foi possível compreender as aproximações entre os dois grupos de acadêmicos, quando partilham elementos do cuidado humano, e distanciamentos, quando não compartilham elementos da prática do cuidado de enfermagem, que estão presentes apenas no grupo de acadêmicos do último período.

Diante desses dados, pode-se identificar a potencial influência de tais representações sobre a prática profissional.

Tendo como base a representação construída pelos acadêmicos iniciantes, pode-se inferir que estes adotam práticas e comportamentos de atenção e afetividade com o próximo e de entrega pela vida do outro, enquanto os acadêmicos concluintes agem conjugando aspectos afetivo-expressivos e técnico-instrumentais e, por isso, buscam conhecer o outro para atender às suas necessidades.

Deste modo, todos os acadêmicos determinam uma forma de realizar o cuidado de enfermagem voltado a atender o cliente, sendo a diferença o objetivo da ação. Para os acadêmicos iniciantes devem ser realizadas com aspectos afetivos, e para os concluintes, pela aplicação do conhecimento científico.

\section{CONSIDERAÇÕES FINAIS}

A dimensão prática do cuidado deve ser baseada na interação que permita o diálogo e o respeito das subjetividades, por isso, quando realizadas, necessitam ser éticas, estéticas, e baseadas em conhecimento científico e pessoal.

Dentre os aspectos da dimensão prática do cuidado de enfermagem, o cuidado humano voltado aos aspectos afetivos foi encontrado nos dois grupos, destacando-se no do primeiro período.

Evidenciou-se que a representação social do cuidado de enfermagem de cada um dos grupos sofreu transformações através da inserção de diferentes elementos de origem acadêmica, necessários ao conhecimento e à prática do cuidado. 
Como exemplo, foi possível indicar: o conhecimento científico e sua aplicação em situações reais e simuladas de cuidado de enfermagem.

Os resultados apontam que tantos os iniciantes quantos os concluintes produzem discursos que veiculam elementos que colaboram para a compreensão do cuidado de enfermagem como uma ação própria do enfermeiro.

Reconhece-se, por fim, que os resultados encontrados conduzem a reflexão sobre a prática e o ensino da enfermagem, já que o currículo de graduação produziu nos alunos mudanças em seus modos de representar o cuidado de enfermagem. Ao acessar as RS dos alunos no início e no final do curso, foi possível, indiretamente, avaliar o processo de ensino-aprendizagem do cuidado de enfermagem. Conhecer os elementos que conformam os conteúdos de RS sobre o cuidado de enfermagem possibilitou identificar necessidades de intervenções pedagógicas, de modo a incluir conteúdos (informações, afetos e atitudes) e experiências que mobilizem tais elementos e os reorganizem no campo para, assim, buscar se elevar cada vez mais a qualidade da assistência e as boas práticas, já que o alcance da TRS está em, justamente, conhecer os saberes sociais que justificam determinadas práticas dos grupos.

\section{REFERÊNCIAS}

1.Vale EG, Pagliuca LMF. Construção de um conceito de cuidado de enfermagem: contribuição para o ensino de graduação. Rev Bras Enferm. $2011 \mathrm{jan} / \mathrm{fev} ;$ 64(1): 106-13.

2.Souza ML, Sartor VVB, Padilha MICS, Prado, ML. 0 cuidado em enfermagem: uma aproximação teórica. Texto\& Contexto Enferm. 2005 abr/jun; 14(2): 266-70.

3.Schoeller SD, Leopardi MT, Ramos FS. Cuidado: eixo da vida, desafio da enfermagem. Rev Enferm UFSM. 2011 jan/abr; 1(1): 88-96

4.Ferreira MA. A comunicação no cuidado: uma questão fundamental na enfermagem. Rev Bras Enferm. 2006 jun; 59(3): 327-30.

5.Silva KL, Sena RR de, Grillo MJC, Horta NC. Formação do enfermeiro: desafios para a promoção da saúde. Esc Anna Nery. 2010 abr/jun; 14(10): 368-76.

6.Souza MHN, PazEPA, Griep RH, Sousa Al, Silva LL, Paixão AR. Experiências de ensino-aprendizagem de estudantes de enfermagem em uma comunidade do município do Rio de Janeiro. Esc Anna Nery. 2006 ago; 10(2): 251-57.

7.Moscovici S. A representação social da psicanálise. Rio de Janeiro: Zahar; 1978

8.Jodelet D. Representações sociais: um domínio em expansão. In: Jodelet D, organizadora. As representações sociais. Rio de Janeiro: Eduerj; 2002. p. 17-44.
9.Fontes CAS, Alvim NAT. A relação humana no cuidado de enfermagem junto ao cliente com câncer submetido à terapêutica antineoplásica. Acta Paul Enferm. 2008 mar; 21 (1): 77-83.

10.Leininger MM. Etnonursing: a research methods with enablers to study the Theory of Culture Care. In: Leininger MM, McFarland MR Culture care diversity and universality: a worldwide nursing theory. Massachusetts: Jones and Bartlett Learning; 2006. p.43-81.

11.Vale EG, Pagliuca LMF, Quirino RHR. Saberes e práxis em enfermagem. Esc Anna Nery. 2009 jan/mar; 13(1): 174-80.

12.Baggio MA, Callegaro GD, Erdmann AL. Relações de "não cuidado" de enfermagem em uma emergência: que cuidado é esse? Esc Anna Nery. [on-line] 2011; jan/mar [citado 2011 maio 20]; 15(1): [aprox.13 telas]. Disponível em http://www.scielo.br

13.Balduino AF, Mantovani MF, Lacerda MR. 0 processo de cuidar de enfermagem ao portador de doença crônica cardíaca. Esc Anna Nery. 2009 abr/jun; 13 (2): 342-51

14.Garcia TR, Nóbrega MML. Processo de enfermagem: da teoria à prática assistencial e de pesquisa. Esc Anna Nery. 2009 jan/mar; 13 (1): 188- 93.

15.Moscovici S. Representações sociais: investigações em psicologia social. Petrópolis(RJ): Vozes; 2007.

\section{NOTA}

aRecorte extraído da dissertação de Mestrado intitulada: "Representaç̃̃es Sociais sobre o cuidado de enfermagem pelos alunos da graduação: implicações para a prática e o ensino de enfermagem.", apresentada ao Programa de Pós-graduação em Enfermagem da Escola de Enfermagem Anna Nery - Universidade Federal do Rio de Janeiro, 2010. Aluna bolsista CNPq (ano 2008-2010). 